aim of this film was to show how a minimal invasive surgical approach is safe and feasible in selected patients.

Description In the video we present the case of a hepato-renal recess isolated recurrence of serous endometrial carcinoma. Patient positioning and port placement were critical to achieve an optimal exposure of the liver 6th segment and the Morison's pouch. An extensive removal of visceral adhesions was performed and the metastasis was then exposed. The tumor infiltrated the liver parenchyma for less than $2 \mathrm{~cm}$. A superficial wedge resection was executed until complete removal of the lesion. The procedure has been performed in 90 minutes. Post-operative course was unremarkable and the patient was discharged on post-operative day 3. The patient was able to resume her oral PARP inhibitor after 10 days. Histopathology report confirmed the metastatic localization of a high-grade serous cancer.

Conclusions Potential benefits of minimally invasive approach include reduced blood loss, less pain, faster recovery and a short interval to chemotherapy. The personalised treatment of recurrent endometrial serous cancer should be guided by the molecular pattern of the disease and by the surgical skills with a multidisciplinary approach. video: https:/www.dropbox.com/ s/glhyzjy7na5101s/MINIMALLY\%20INVASIVE\%20SECONDARY \%20CYTOREDUCTIVE\%20SURGERY\%20FOR\%20HEPATORENAL \%20RECESS\%20ISOLATED\%20RECURRENCE\% 20OF\%20SEROUS\%20ENDOMETRIAL\%20CANCER\%20IN \%20BRCA1\%20MUTATED\%20PATIENT.mp4? dl=0

\section{SF022/\#580 IDENTIFICATION OF INGUINAL SENTINEL LYMPH NODES IN RECURRENT VULVAR MELANOMA}

D Knigin*, T Perri, G Levin, B Brandt, L Kogan. Hadassah Medical Center, Gynecologic Oncology, Jerusalem, Israel

\subsection{6/ijgc-2021-IGCS.66}

Introduction Vulvar melanoma has a high propensity for lymphatic spread to inguinal lymph nodes. Groin sentinel lymph node biopsy is considered a part of the management of primary vulvar melanoma. Yet, the role of performing sentinel lymph node biopsy in the management of patients with negative nodes in primary surgery, who develop recurrent vulvar melanoma, is less clear than in the case of primary tumors. Nevertheless, lymph nodes status in recurrent disease can provide valuable information to guide further therapy. Sentinel mapping in a previously scarred area is challenging and the most suitable mapping technique has not been determined. In this video, we demonstrate the technical feasibility of repeat sentinel lymph nodes biopsy for recurrent vulvar melanoma using intraoperative fluorescent indocyanine green (ICG) detection.

Description The video demonstrates ICG mapping technique in recurrent vulvar melanoma. The injection site and ICG positive nodes are shown. Implication: Sentinel mapping in recurrent vulvar melanoma with ICG tracer technique is feasible, even in previously dissected groins.

\section{SF023/\#590 VULVECTOMY- AN OPERATIVE PROCEDURE FOR CA VULVA}

R Pratima*, R Sekhon. Rajiv Gandhi Cancer Institute and Rearch Center, Gynaecology Oncology, New Delhi, India

10.1136/ijgc-2021-IGCS.67
Introduction Increasing knowledge and understanding of the disease has allowed surgical procedures for the treatment of carcinoma of the vulva to become more conservative and individualized to each patient. The exact procedure used depends upon the site, size, and histologic features of the tumor.

Description Preoperative preparation - All women require explanation and counseling about the procedure. General anesthesia is administered. The patient is positioned in lithotomy. The skin is prepared and draped. The patient is examined and the skin to be incised is marked with a pen. A urethral catheter is inserted into the bladder Skin incision is begun with scalpel and the dissection is taken through the subcutaneous fat to the deep fascia and pubic ramus until the intended vaginal resection margin is reached with scalpel, scissors and diathermy. The dissection is carried down toward the clitoral attachments by sweeping the specimen off the periosteum of the pubic bones conserving the deep fascia until the clitoral attachments are reached. The suspensory ligament of the clitoris is clamped, divided, and ligated. The urethro-vaginal incision is now made circumferentially, ensuring that the required margin around the tumor is maintained. The tip of a scalpel Kelly forceps is passed through the specimen in the midline to isolate the crura divide and then suture ligated with absorbable sutures. The specimen is detached completely, and hemostasis is secured. The wound closed primarily.

Conclusion Adequate surgical resection with microscopic tumor-free margin should be the key concern. Oncological resection should be equated with functional outcome.

\section{E-Posters}

\section{E-Poster Viewing}

\section{EPV001/\#119 SCREENING AND IDENTIFICATION OF NOVEL CHEMOTHERAPY AGENTS IN PLATINUM- RESISTANT OVARIAN CANCER}

${ }^{1} \mathrm{D}$ Schweer*, ${ }^{2} \mathrm{~J}$ Mccorkle, ${ }^{2} \mathrm{~J}$ Rohr, ${ }^{2} \mathrm{O}$ Tsodikov, ${ }^{1} \mathrm{~F}$ Ueland, ${ }^{2} \mathrm{~J}$ Kolesar. ${ }^{1}$ University of Kentucky, Obstetrics and Gynecology, Lexington, USA; ${ }^{2}$ University of Kentucky, Pharmaceutical Sciences, Lexington, USA

\subsection{6/ijgc-2021-IGCS.68}

Objectives To screen the efficacy of potential chemotherapeutics against platinum-sensitive \& resistant ovarian cancer cell lines.

Methods We performed in-vitro screening on mithramycin, an antineoplastic antibiotic; telaglenastat, a glutaminase inhibitor; savolitinib, a c-met tyrosine kinase inhibitor; and AMG-232, an MDM2 inhibitor. We tested all agents against a platinumresistant cell line (OVCAR3) and a platinum-sensitive line (CAOV3). Additionally, we tested mithramycin against UWB1.289, a BRCA mutant, and an induced platinum-resistant UWB1.289 line. DMSO and cisplatin were the negative and positive controls, respectively, and we performed all experiments in triplicate. Cell viability was determined by measuring cellular ATP content.

Results The IC50 values of mithramycin ranged from 42.4 to $65.5 \mathrm{nM}$. Cisplatin IC50 values ranged from a median of 2067 to $7267 \mathrm{nM}$. The IC50 values of telaglenastat, savolitinib, and AMG-232 did not reach a level of $10 \mu \mathrm{m}$ in any of the 
tested cell lines. Table 1 . IC50 values of agents sorted by cell lines. N/A indicating value not achieved at threshold of $10 \mathrm{um}$. Conclusions Platinum-resistant ovarian cancer has a poor prognosis highlighting the need for new therapeutic modalities. Neither telaglenastat, savolitinib, nor AMG-232 exhibit any appreciable cytotoxicity; however, mithramycin demonstrates

\begin{tabular}{lcccc}
\multicolumn{5}{c}{ Abstract EPV001/\#119 Table 1} \\
\hline & $\begin{array}{c}\text { CAOV3 } \\
(\mathbf{n}=3)\end{array}$ & $\begin{array}{c}\text { UWB1.289 } \\
(\mathrm{n}=3)\end{array}$ & $\begin{array}{c}\text { OVCAR3 } \\
(\mathrm{n}=3)\end{array}$ & $\begin{array}{c}\text { UWB1.289-Platinum } \\
\text { Resistant(n=3) }\end{array}$ \\
\hline Cisplatin(nm) & 2733 & $2067 \pm 416$ & 4733 & $7267 \pm 2572$ \\
& \pm 208 & & \pm 1250 & \\
Mithramycin & $42.4 \pm 8$ & $57.9 \pm 46$ & $65.5 \pm 38$ & $62.2 \pm 54$ \\
$(\mathrm{~nm})$ & $\mathrm{N})$ & $\mathrm{N} / \mathrm{A}$ & $\mathrm{N} / \mathrm{A}$ & $\mathrm{N} / \mathrm{A}$ \\
Teleganestat & $\mathrm{N} / \mathrm{A}$ & $\mathrm{N} / \mathrm{A}$ & $\mathrm{N} / \mathrm{A}$ & $\mathrm{N} / \mathrm{A}$ \\
Savolitinib & $\mathrm{N} / \mathrm{A}$ & $\mathrm{N} / \mathrm{A}$ & $\mathrm{N} / \mathrm{A}$ & $\mathrm{N} / \mathrm{A}$ \\
AMG-232 & $\mathrm{N} / \mathrm{A}$ & & & \\
\hline
\end{tabular}

cytotoxicity in the low nanomolar range in several representative ovarian cancer cell lines, including two platinum-resistant lines. The potential therapeutic benefit of mithramycin warrants further preclinical evaluation.

\section{EPV002/\#308 METABOLOMIC ADAPTATIONS ASSOCIATED WITH CHEMORESISTANCE IN OVARIAN CANCER CELL LINES}

${ }^{1} \mathrm{~L}$ Corey ${ }^{*},{ }^{2} \mathrm{G}$ Mor, ${ }^{1} \mathrm{~L}$ Matherly, ${ }^{3} \mathrm{~J}$ Jiang, ${ }^{3} \mathrm{Y} Y$ Yue, ${ }^{4} \mathrm{~N}$ Tiwari, ${ }^{1} \mathrm{Z}$ Hou, ${ }^{1} \mathrm{Y}$ You, ${ }^{3} \mathrm{~J} \mathrm{Li},{ }^{5} \mathrm{~S}$ Kim, ${ }^{1} \mathrm{R}$ Rattan, ${ }^{1} \mathrm{~A}$ Alvero, ${ }^{6} \mathrm{R}$ Gogoi. ${ }^{1}$ Wayne State University, Oncology, Detroit, USA; ${ }^{2}$ Wayne State University, Obstetrics and Gynecology, Detroit, USA; ${ }^{3}$ Wayne State University, Metabolomics, Detroit, USA; ${ }^{4}$ Henry Ford Health Systems, Oncology, Detroit, USA; ${ }^{5}$ Wayne State University, Biostatistics, Detroit, USA; ${ }^{6}$ Wayne State University/Karmanos Cancer Institute. Division of Gynecologic Oncology, Gyn Oncology, Northville, USA

\subsection{6/ijgc-2021-IGCS.69}

Objectives Over $80 \%$ of ovarian cancer (OC) patients will experience relapse after an initial response to platinum-based chemotherapy. Acquisition of metabolomic adaptations is
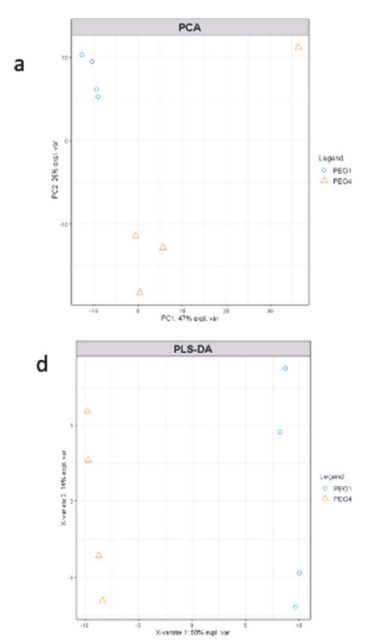
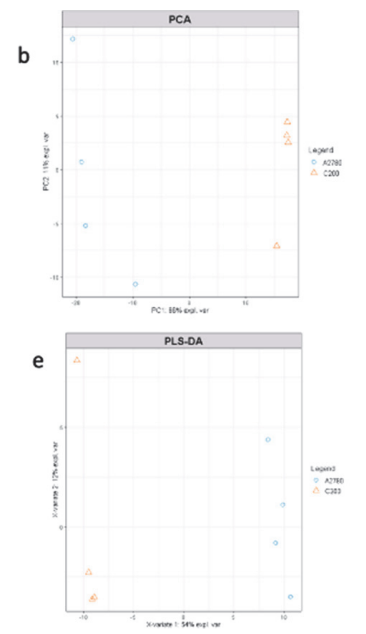
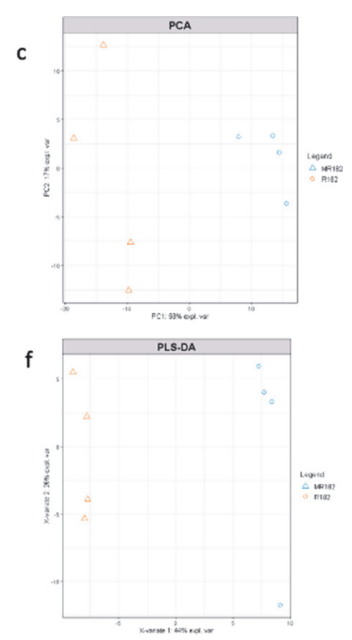

Abstract EPV002/\#308 Figure 1 PCA of a. PE01 vs PE04, b. A2780 vs C200, c. MR182 vs R182; PLS-DA of d. PE01 vs PE04, e. A2780 vs C200, f. MR182 vs R182
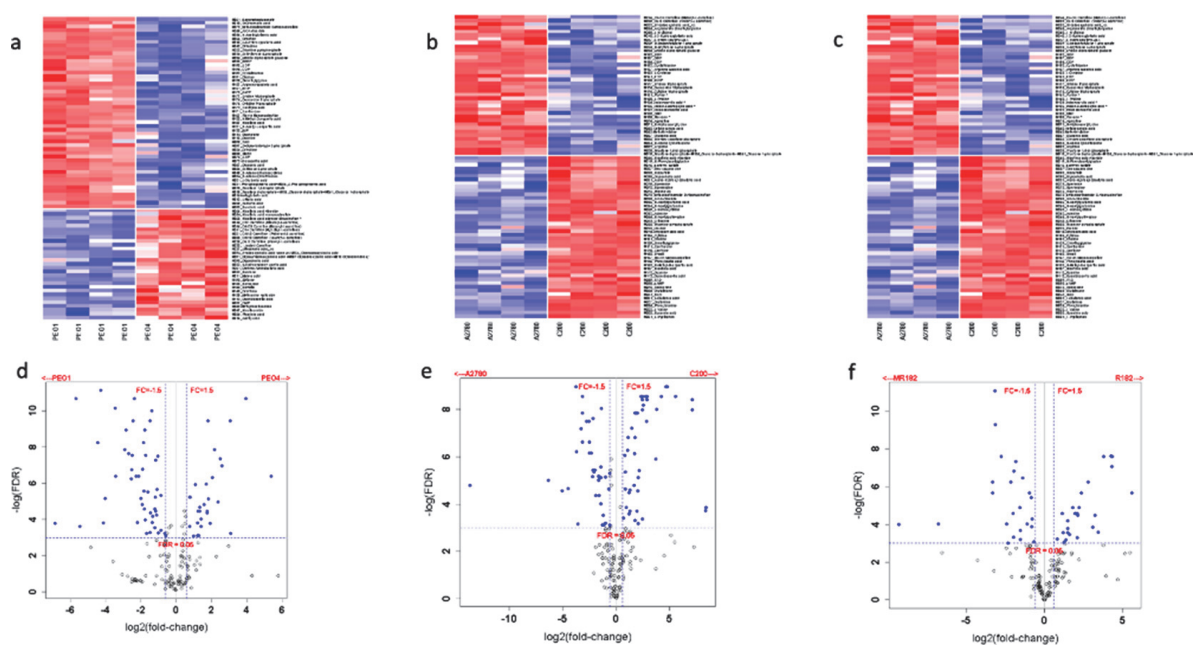

Abstract EPV002/\#308 Figure 2 Heat map of a. PE01 vs PEO4, b. A2780 vs C200, c. MR182 vs R182; Volcano plot with FDR $\leq 5 \%$ and absoulute fold-change $\geq 1.5$ of $d$. PE01 vs PE04, e. A2780 vs C200, f. MR182 vs R182 